\title{
Prompt photon photoproduction at HERA within the framework of the quark Reggeization hypothesis
}

\author{
V.A. Saleev* \\ Samara State University, Ac. Pavlov St. 1, 443011 Samara, Russia
}

\begin{abstract}
We study the inclusive production of isolated prompt photons within the framework of the quasi-multi-Regge-kinematic approach, applying the quark Reggeization hypothesis. We describe accurately and without free parameters the transverse momentum and pseudorapidity spectra of prompt photons in the inclusive photoproduction at the HERA Collider. It is shown that the main mechanism of the inclusive prompt photon production in the $\gamma p$ collisions is the fusion of a Reggeized quark (antiquark) from the proton and a collinear antiquark (quark) from the photon into a photon, via the effective Reggeon-quark-gamma vertex. The fragmentation of the quark, which is produced via the gamma-Reggeon-quark and quark-Reggeon-quark vertices, into a photon is strongly suppressed by the isolation cone condition and it gives a significant contribution in the region of a large negative pseudorapidity only. At the stage of numerical calculations we use the Kimber-Martin-Ryskin prescription for unintegrated quark and gluon distribution functions, with the following collinear parton densities as input: MRST for a proton and GRV for a photon.
\end{abstract}

PACS numbers: 12.38.-t,13.60.Hb,13.85.-t

*Electronic address: saleev@ssu.samara.ru 


\section{INTRODUCTION}

It is well known that studies of production of photons with large transverse momenta producing in the hard interaction between photon and parton or between two partons in $\gamma p$ collisions, so-called prompt photon production, provide precision tests of perturbative quantum chromodynamics (QCD) as well as information on the parton densities within a proton and a photon.

Also, these studies are our potential for the observation of a new dynamical regime, namely the high-energy Regge limit, which is characterized by the following condition $\sqrt{S}>>\mu>>\Lambda_{Q C D}$, where $\sqrt{S}$ is the total collision energy in the center of mass reference frame, $\Lambda_{Q C D}$ is the asymptotic scale parameter of QCD, $\mu$ is the typical energy scale of the hard interaction. At this high-energy limit, the contribution from the partonic subprocesses involving $t$-channel parton (quark or gluon) exchanges to the production cross section can become dominant. In the region under consideration, the transverse momenta of the incoming partons and their off-shell properties can no longer be neglected, and we deal with "Reggeized" $t$-channel partons.

We consider the quasi-multi-Regge kinematics (QMRK) approach [1], which is similar to the $k_{T}$-factorization approach [2] conceptually, as the theoretical framework for this kind of high-energy phenomenology. Our previous analysis of charmonium and bottomonium production at the Fermilab Tevatron and DESY HERA Colliders [3], and the study of prompt photon production at the Fermilab Tevatron [4], using the high-energy factorization scheme have shown the appreciation of the method even at the leading order (LO) in the strong-coupling constant $\alpha_{s}$ in compare with the experimental data. We have found that the essential features produced by the high-energy factorization scheme at the LO are being at the next-to-leading (NLO) in the conventional collinear parton model.

Contrary to the $k_{T}$-factorization scheme, based on the well-known prescription for offshell $t$-channel gluons [2], the QMRK approach seems to be more proper theoretically [5]. In this approach we work with gauge invariant amplitudes and use the factorization hypothesis, which is proved in the leading-logarithmic-approximation (LLA). In the QMRK approach we can do calculationSs with Reggeized quarks [4], that presents an open question in the $k_{T}$-factorization approach, following which we can operate correctly with off-shell gluons only. Recently it was shown also, that the calculation in the NLO in strong coupling 
constant within the framework of QMRK approach can be done [6] .

In this paper we study the inclusive photoproduction of prompt photon within the framework of the QMRK approach, applying the quark and gluon Reggeization hypothesis [1, 7]. We describe accurately and without free parameters the transverse momentum and pseudorapidity spectra of prompt photons in the inclusive photoproduction at the HERA Collider [8, 9]. It is shown that the main mechanism of the inclusive prompt photon production in the $\gamma p$ collisions is the fusion of a Reggeized quark (antiquark) from the proton and a collinear antiquark (quark) from the photon into a photon, via the effective Reggeon-quark-gamma vertex. The fragmentation of the quark, which is produced via the gamma-Reggeon-quark and quark-Reggeon-quark vertices, into a photon is strongly suppressed by the isolation cone condition and it gives a significant contribution in the region of a large negative pseudorapidity only. At the stage of numerical calculations we use the Kimber-Martin-Ryskin prescription for unintegrated quark and gluon distribution functions [10], with the following collinear densities as input: MRST for a proton [11] and GRV for a photon [12].

This paper is organized as follows. In Sec. II, the relevant Reggeon-Reggeon-particle and particle-Reggeon-particle effective vertices are presented and discussed. In Sec. III, we describe the inclusive photon photoproduction at the HERA Collider. In Sec. IV, we compare our approach with the similar calculations in the $k_{T}$-factorization scheme. In Sec. V, we summarize our conclusions.

\section{BASIC FORMALISM}

In the phenomenology of strong interactions at high energies, it is necessary to describe the QCD evolution of the parton distribution functions of the colliding particles starting with some scale $\mu_{0}$, which controls a nonperturbative regime, to the typical scale $\mu$ of the hard-scattering processes, which is typically of the order of the transverse mass $M_{T}=$ $\sqrt{M^{2}+\left|\mathbf{p}_{T}\right|^{2}}$ of the produced particle with mass $M$ and transverse momentum $\mathbf{p}_{T}$. In the region of very high energies, in the so-called Regge limit, the typical ratio $x=\mu / \sqrt{S}$ becomes very small, $x \ll 1$. This leads to large logarithmic contributions of the type $\left[\alpha_{s} \ln (1 / x)\right]^{n}$ in the resummation procedure, which is described by the BFKL evolution equation [13] or other BFKL-like ones for an unintegrated gluon (quark) distribution functions $\Phi_{g, q}\left(x,\left|\mathbf{q}_{T}\right|^{2}, \mu^{2}\right)$. Correspondingly, in the QMRK approach [1], the initial-state $t$-channel gluons and quarks 
are considered as Reggeons, or as Reggeized gluons $(R)$ and as Reggeized quarks $(Q)$. They are off-mass shell and carry finite transverse two-momenta $\mathbf{q}_{T}$ with respect to the hadron beam from which they stem.

Recently, in Ref. [14], the Feynman rules for the effective theory based on the non-Abelian gauge-invariant action [5] were derived for the induced and some important effective vertices. However, these rules include processes with Reggeized gluons in the initial state only. In case of $t$-channel quark exchange processes such rules are absent today, in spite of that the gauge-invariant action for Reggeized quarks is known [15], and it is needed to construct effective vertices with the Reggeized quarks, using QMRK approach prescriptions, every time from the beginning. Of course, a certain set of Reggeon-Reggeon-particle effective vertices is known, for example, for the transitions $R R \rightarrow g$ [16], $Q \bar{Q} \rightarrow g$ [7] and $\gamma^{*} Q \rightarrow q$ [4].

Roughly speaking, the Reggeization of amplitudes is a trick that offers an opportunity to take into account efficiently large radiation corrections to the processes under Regge limit condition outside the collinear approximation. The particle Reggeization is known in high energy quantum electrodynamics (QED) for electrons only [17] and for gluon and quarks in QCD [7, 13].

The effective vertex $C_{\bar{Q} Q}^{g}\left(q_{1}, q_{2}\right)$, which describes the production of a single gluon with momentum $k(g)=q_{1}(Q)+q_{2}(\bar{Q})$ in the Reggeized quark and Reggeized antiquark annihilation was obtained in Ref. [7, 19] and it can be presented, omitting the color and Lorentz indexes in the left side, as follows:

$$
C_{\bar{Q} Q}^{g}\left(q_{1}, q_{2}\right)=D_{a}^{\mu}\left(q_{1}, q_{2}\right)=-g_{s} T^{a}\left(\gamma^{\mu}-\frac{2 P_{1}^{\mu}}{x_{2} S} \hat{q_{2}}-\frac{2 P_{2}^{\mu}}{x_{1} S} \hat{q_{1}}\right),
$$

where $P_{1,2}$ are colliding hadron momenta, $P_{(1,2)}=E_{(1,2)}(1, \overrightarrow{0}, \pm 1)$ and $S=2\left(P_{1} P_{2}\right)=$ $4 E_{1} E_{2}$. The Reggeized quark momenta are $q_{1}=x_{1} P_{1}+q_{1 T}, q_{2}=x_{2} P_{2}+q_{2 T}$, and $q_{(1,2) T}=\left(0, \vec{q}_{(1,2) T}, 0\right)$. It is obvious that this vertex satisfies the gauge-invariant condition $D_{a}^{\mu}\left(q_{1}, q_{2}\right) k_{\mu}=0$.

Like the gluon production, the effective vertex for photon production can be written in the form:

$$
C_{\bar{Q} Q}^{\gamma}\left(q_{1}, q_{2}\right)=F^{\mu}\left(q_{1}, q_{2}\right)=-e e_{q}\left(\gamma^{\mu}-\frac{2 P_{1}^{\mu}}{x_{2} S} \hat{q_{2}}-\frac{2 P_{2}^{\mu}}{x_{1} S} \hat{q_{1}}\right),
$$

where $e=\sqrt{4 \pi \alpha}$ is the electromagnetic coupling constant, $e_{q}$ is the quark electric charge. 
The effective vertex, which describes the production of a quark with momentum $k=$ $q_{1}\left(\gamma^{\star}\right)+q_{2}(Q)$ in a virtual photon collision with a Reggeized quark can be presented as follows [4]:

$$
C_{\gamma Q}^{q}\left(q_{1}, q_{2}\right)=G^{\mu}\left(q_{1}, q_{2}\right)=-e e_{q}\left(\frac{q_{1}^{2}}{q_{1}^{2}+q_{2}^{2}} \gamma^{\mu}-\frac{2 k^{\mu}}{q_{1}^{2}+q_{2}^{2}} \hat{q_{2}}+\frac{2 q_{2}^{2} x_{2} P_{2}^{\mu}}{\left(q_{1}^{2}+q_{2}^{2}\right)^{2}} \hat{q_{2}}\right),
$$

where $q_{2}=x_{2} P_{2}+q_{2 T}$ is the Reggeized quark four-momentum and the gauge invariance condition is given by $G^{\mu}\left(q_{1}, q_{2}\right) q_{1 \mu}=0$. For a mass-shell photon the vertex (3) can be presented in the form:

$$
G^{\mu}\left(q_{1}, q_{2}\right)=2 e e_{q} \frac{\hat{q}_{2 T} q_{2 T}^{\mu}}{q_{2}^{2}} .
$$

The effective Reggeon-Reggeon-particle vertex, which describes the production of a quark with momentum $k(q)=q_{1}(Q)+q_{2}(R)$ in the Reggeized quark and Reggeized gluon collision can be presented as follows:

$$
C_{R Q}^{q}\left(q_{1}, q_{2}\right)=V\left(q_{1}, q_{2}\right)=-g_{s} T^{a} \hat{\varepsilon}_{T}\left(q_{2}\right)
$$

where $\hat{\varepsilon}_{T}\left(q_{2}\right)=\gamma_{\mu} \varepsilon^{\mu}\left(q_{2}\right)$ and $\varepsilon^{\mu}\left(q_{2}\right)=\frac{q_{2 T}^{\mu}}{\left|\vec{q}_{2 T}\right|}$.

Let us note that contrary to the definition used in Ref. [19], we do not include quark spinors in our equations for the effective vertices (11)-(15).

The relevant squared matrix elements can be presented as follows:

$$
\begin{aligned}
\overline{|M(Q \bar{Q} \rightarrow \gamma)|^{2}} & =\frac{4}{3} \pi \alpha e_{q}^{2}\left(t_{1}+t_{2}\right), \\
\overline{|M(R Q \rightarrow q)|^{2}} & =\frac{2}{3} \pi \alpha_{s}\left(t_{1}+t_{2}+2 \sqrt{t_{1} t_{2}} \cos \phi\right), \\
\overline{|M(\gamma Q \rightarrow q)|^{2}} & =8 \pi \alpha e_{q}^{2} t_{2}, \\
\overline{|M(R R \rightarrow g)|^{2}} & =\frac{3}{2} \pi \alpha_{s}\left(t_{1}+t_{2}+2 \sqrt{t_{1} t_{2}} \cos \phi\right),
\end{aligned}
$$

where $t_{1}=\vec{q}_{1 T}^{2}, t_{2}=\vec{q}_{2 T}^{2}$, and $\phi$ is the azimuthal angle between $\vec{q}_{1 T}$ and $\vec{q}_{2 T}$.

\section{INCLUSIVE PROMPT PHOTON PHOTOPRODUCTION AT HERA}

In this part we consider the inclusive prompt photon production at the HERA Collider in the region of a small exchange photon virtuality $Q^{2}<1 \mathrm{GeV}^{2}$, i.e. in the photoproduction processes. Working in the LO QMRK approach we describe data applying the quark and gluon Reggeization hypothesis. There are two main mechanisms of prompt photon production in this approach: the production of direct photons and the production of photons via 
fragmentation. In the first case, photons are produced directly via fusion of a Reggeized quark and a Reggeized antiquark, which stem from a proton (photon) and a photon (proton):

$$
Q_{\gamma}\left(\bar{Q}_{\gamma}\right)+\bar{Q}_{p}\left(Q_{p}\right) \rightarrow \gamma
$$

In the second case, photons are produced in the quark or gluon fragmentation into the photon, which is described by the parton to photon fragmentation functions $D_{q \rightarrow \gamma}\left(z, \mu^{2}\right)$ and $D_{g \rightarrow \gamma}\left(z, \mu^{2}\right)[20]$. In the discussed here task the gluon fragmentation into the photon is unimportant and we will take into account the quark(antiquark) fragmentation only:

$$
\begin{aligned}
& \gamma+Q_{p}\left(\bar{Q}_{p}\right) \rightarrow q(\bar{q}) \rightarrow \gamma, \\
& Q_{\gamma}\left(\bar{Q}_{\gamma}\right)+R_{p} \rightarrow q(\bar{q}) \rightarrow \gamma, \\
& R_{\gamma}+Q_{p}\left(\bar{Q}_{p}\right) \rightarrow q(\bar{q}) \rightarrow \gamma
\end{aligned}
$$

The factorization formulas for the production cross section of the direct photons (10) can be written as follows:

$$
\begin{aligned}
d \sigma^{d i r}(e N \rightarrow \gamma X)= & \sum_{q, \bar{q}} \int d y \int d x_{1} \int \frac{d^{2} q_{1 T}}{\pi} \int d x_{2} \int \frac{d^{2} q_{2 T}}{\pi} G_{\gamma / e}(y) \Phi_{q / \gamma}\left(x_{1}, t_{1}, \mu^{2}\right) \times \\
& \times \Phi_{\bar{q} / p}\left(x_{2}, t_{2}, \mu^{2}\right) d \hat{\sigma}(Q \bar{Q} \rightarrow \gamma) .
\end{aligned}
$$

In the case of fragmentation production mechanism we have two formulas for the "direct" (11) and the "resolved" (12]13) contributions:

$$
\begin{gathered}
d \sigma^{f r, d i r}(e N \rightarrow \gamma X)=\sum_{q, \bar{q}} \int d z \int d y \int d x_{2} \int \frac{d^{2} q_{2 T}}{\pi} G_{\gamma / e}(y) \times \\
\quad \times \Phi_{g / p}\left(x_{2}, t_{2}, \mu^{2}\right) d \hat{\sigma}(\gamma Q \rightarrow q) D_{q \rightarrow \gamma}\left(z, \mu^{2}\right), \\
d \sigma^{f r, r e s}(e N \rightarrow \gamma X)=\sum_{\substack{q, \bar{q}\\
}} \int d z \int d y \int d x_{1} \int \frac{d^{2} q_{1 T}}{\pi} \int d x_{2} \int \frac{d^{2} q_{2 T}}{\pi} G_{\gamma / e}(y) \times \\
\\
\times \Phi_{q / \gamma}\left(x_{1}, t_{1}, \mu^{2}\right) \Phi_{g / p}\left(x_{2}, t_{2}, \mu^{2}\right) d \hat{\sigma}(Q R \rightarrow q) D_{q \rightarrow \gamma}\left(z, \mu^{2}\right) .
\end{gathered}
$$

Here $\Phi_{q, g / \gamma}\left(x_{1}, t_{1}, \mu^{2}\right)$ is the unintegrated quark or gluon distribution in a photon, which is calculated using KMR[10] prescription, the same as the unintegrated quark or gluon distribution in a proton $\Phi_{q, g / p}\left(x_{2}, t_{2}, \mu^{2}\right) ; G_{\gamma / e}(y)$ denotes the photon flux integrated over $Q^{2}$ from the kinematic limit of $Q_{\min }^{2}=m_{e}^{2} y^{2} /(1-y)$ to the upper limit $Q_{\max }^{2}=1 \mathrm{GeV}^{2}$; $y=W^{2} / S_{e N}, W^{2}=2\left(P_{\gamma} P_{N}\right), S_{e N}=2\left(P_{e} P_{N}\right)=4 E_{N} E_{e}, E_{N}$ and $E_{e}$ are the proton and 
electron energies in the laboratory frame. We use the following collinear densities as input: MRST [11] for a proton and GRV [12] for a photon. The exact formula for $G_{\gamma / e}(y)$ is taken as follows:

$$
G_{\gamma / e}(y)=\frac{\alpha}{2 \pi}\left[\frac{1+(1-y)^{2}}{y} \log \frac{Q_{\max }^{2}}{Q_{\min }^{2}}+2 m_{e}^{2} y\left(\frac{1}{Q_{\min }^{2}}-\frac{1}{Q_{\max }^{2}}\right)\right] .
$$

To calculate transverse momentum and pseudorapidity spectra in the photoproduction of the direct photon we use the following formula, which is obtained from (14):

$$
\begin{aligned}
\frac{d \sigma}{d p_{T} d \eta}(e N \rightarrow \gamma X)= & \frac{1}{p_{T}^{3}} \sum_{q, \bar{q}} \int d y \int d \phi_{1} \int d t_{1} G_{\gamma / e}(y) \Phi_{\bar{q}, q / \gamma}\left(x_{1}, t_{1}, \mu^{2}\right) \times \\
& \times \Phi_{q, \bar{q} / p}\left(x_{2}, t_{2}, \mu^{2}\right) \overline{|M(Q \bar{Q} \rightarrow \gamma)|^{2}}
\end{aligned}
$$

where $p=\left(p_{0}, \mathbf{p}_{T}, p_{z}\right)$ is the produced photon four-momentum, $P_{e}=E_{e}(1, \mathbf{0},-1), P_{N}=$ $E_{N}(1, \mathbf{0}, 1)$,

$$
x_{1}=\frac{p_{0}-p_{z}}{2 E_{e} y}, \quad x_{2}=\frac{p_{0}+p_{z}}{2 E_{N}}, \quad t_{2}=t_{1}-2 p_{T} \sqrt{t_{1}} \cos \left(\phi_{1}\right)+p_{T}^{2} .
$$

In case of the fragmentation production mechanism the master formulas can be obtained from (16), and (17) and they have the following forms:

$$
\begin{aligned}
\frac{d \sigma}{d p_{T} d \eta}(e N \rightarrow \gamma X) & =\frac{2 \pi}{p_{T}^{3}} \sum_{q, \bar{q}} \int d y G_{\gamma / e}(y) \Phi_{q, \bar{q} / p}\left(x_{2}, t_{2}, \mu^{2}\right) \times \\
& \times z^{3} D_{q \rightarrow \gamma}\left(z, \mu^{2}\right) \overline{\mid M\left(\left.\gamma Q \rightarrow q\right|^{2}\right.}
\end{aligned}
$$

where

$$
\begin{gathered}
z=\frac{p_{0}-p_{z}}{2 y E_{e}}, \quad x_{2}=\frac{p_{0}+p_{z}}{2 z E_{N}}, \quad t_{2}=\frac{p_{T}^{2}}{z^{2}} \\
\frac{d \sigma}{d p_{T} d \eta}(e N \rightarrow \gamma X)=\frac{1}{p_{T}^{3}} \sum_{q, \bar{q}} \int d y \int d \phi_{1} \int d t_{1} \int d z G_{\gamma / e}(y) \Phi_{q, \bar{q}}^{\gamma}\left(x_{1}, t_{1}, \mu^{2}\right) \Phi_{g}^{p}\left(x_{2}, t_{2}, \mu^{2}\right) \times \\
\times z^{2} D_{q \rightarrow \gamma}\left(z, \mu^{2}\right) \overline{\mid M\left(\left.Q R \rightarrow q\right|^{2}\right.}
\end{gathered}
$$

where

$$
x_{1}=\frac{p_{0}-p_{z}}{2 y z E_{e}}, \quad x_{2}=\frac{p_{0}+p_{z}}{2 z E_{N}}, \quad t_{2}=t_{1}-2 \frac{p_{T}}{z} \sqrt{t_{1}} \cos \left(\phi_{1}\right)+\frac{p_{T}^{2}}{z^{2}} .
$$

The requirement of particle Reggeization demands fulfilment of the conditions $x_{1,2} \ll 1$ for partons both from a proton and a photon in the discussed here processes. As example, in case of the direct photon production we can write:

$$
x_{1}=\frac{p_{T} e^{-\eta}}{2 y E_{e}} \quad \text { and } \quad x_{2}=\frac{p_{T} e^{\eta}}{2 E_{N}},
$$


where for the ZEUS [8] and H1 [9] measurements: $E_{e}=27.5 \mathrm{GeV}, E_{N}=820(920) \mathrm{GeV}$, $|\eta| \leq 1,5<p_{T}<10 \mathrm{GeV}$ and $0.2<y<0.7(0.9)$. If we take the fixed median values: $y=0.5, \eta=0, p_{T}=7.5 \mathrm{GeV}$ and $E_{N}=920 \mathrm{GeV}$, we obtain that $x_{1} \simeq 0.25$ and $x_{2} \simeq 0.004$. In such a way, the particle Reggeization should be used for partons from a proton only, and partons from a photon have been considered in the collinear approximation, as usually in the conventional parton model.

In that case all master formulas can be rewrited using the following prescriptions:

$$
\begin{aligned}
& \int d t_{1} \Phi_{q / \gamma}\left(x_{1}, t_{1}, \mu^{2}\right) \Rightarrow x_{1} F_{q / \gamma}\left(x_{1}, \mu^{2}\right), \\
& \int d \phi_{1} \Rightarrow 2 \pi
\end{aligned}
$$

where $F_{q / \gamma}\left(x_{1}, \mu^{2}\right)$ is the collinear parton density in a photon [12], and we need to take $t_{1}=0$ in the all kinematic relations and in the squared matrix elements.

To compare the theoretical predictions and the data we need to take into account carefully kinematic conditions and cuts of the discussed experiments [8, 9]. The special attention should be attracted to the isolation cone condition $E_{T}^{c o n e}<0.1 E_{T}[9]$, where $E_{T}$ is the total transverse energy of a jet with the detected photon, $E_{T}^{c o n e}$ is the non-photon energy in this jet. It is obvious that the isolation cone condition needs the inequality $0.9<z<1$ to be fulfilled in the case of fragmentation production mechanism. This cut on the fragmentation parameter $z$ strongly suppresses the contribution of the fragmentation production.

However, the isolation cone condition should be taken into account and in the production of direct photons too. The measured transverse energy $E_{T}$ includes additional non-photon part which is order of $E_{T}^{\text {cone }}[9]$, thus we can put $p_{T}=E_{T}-E_{T}^{c o n e} \simeq 0.9 E_{T}$, if the theoretical prediction corresponds to the photon transverse momentum $p_{T}$.

We compare the results of our calculations with the data from ZEUS [8] and H1 [9] Collaborations. Figures (11)-(4) demonstrate an agreement between the theory and the data, especially for the transverse-energy spectra. We use the following notation for the curves: 1 is the resolved production of the direct photons in the subprocesses (10); 2 is the direct production via the fragmentation in the subprocesses (11); 3 is the resolved production via the fragmentation in the subprocesses (12) and (13); 4 is the sum of all contributions.

In the transverse energy distribution the mechanism of the direct photon production (curve 1) is dominant at the all $E_{T}^{\gamma}$ and the fragmentation contribution (curves 2 and 3 ) is only about $5 \%$ of the first one, that is in accordance to the previous calculations in the NLO 
of the collinear parton model [21].

The fragmentation production mechanism gives significant contribution only at the large negative values of $\eta$ in the pseudorapidity distribution. We see that in this region the $\eta$-spectrum can be described correctly if we take into account both the direct (curve 1) and fragmentation (curves 2 and 3) mechanisms. We have obtained that the data lie slightly higher our predictions in case of the ZEUS measurements at all values of pseudorapidity, see Fig.(3). In case of the $\mathrm{H} 1$ measurements the small disagreement is observed in the central region of pseudorapidity $(\eta \approx 0)$ only, see Fig.(4).

The small visible disagreement for the $\eta$-spectra should be explained that we do not use in our calculation the cone isolation condition in the pseudorapidity and azimuthal angle plane

$$
\left(\eta_{j}-\eta\right)^{2}+\left(\phi_{j}-\phi\right)^{2} \leq R
$$

where $R=1$ [8, 9], $\eta_{j}$ and $\phi_{j}$ are the pseudorapidity and azimuthal angle of a hadron from the detected photon jet. Contrary to the collinear parton model calculation, where the LO processes are $2 \rightarrow 2(\gamma q \rightarrow \gamma q, g q \rightarrow \gamma q, \ldots)$, the LO processes in the QMRK approach are $2 \rightarrow 1(Q \bar{Q} \rightarrow \gamma, \ldots)$. The all quark and gluon jets are absorbed in the unintegrated distribution functions of a proton and a photon. Thus, our prediction corresponds correctly the production of "pure" direct photons and it is unclear how to take into account the cone isolation condition (21). Comparing the theoretical predictions and the data we should take into account that the ZEUS and H1 data contain arbitrary small part $(\sim 10 \%)$ of the non-photon events.

\section{DISCUSSION}

In this section, we compare our results with the previous study in the $k_{T}$-factorization scheme, which is similar to the QMRK approach.

The calculation of the inclusive prompt photon production at the HERA Collider was performed using off-shell amplitudes involving initial quarks in the Ref. [22]. In that paper, a Compton process of the off-shell quark - photon scattering $q^{\star}+\gamma \rightarrow q+\gamma$ was considered as the LO direct photon production process. We especially used here different denotations for the Reggeized particles $(Q, R)$ and off-shell particles $\left(q^{\star}, g^{\star}\right)$. It means that in Ref. [22], the conventional QCD vertices are used and the authors obtained gauge uninvariant am- 
plitudes. The gauge invariant condition for amplitudes controls the correct $k_{T}$ dependence of the relevant cross sections. The arbitrary inclusion of the $k_{T}$ effects in the kinematics only, without the using of the correct vertices for Reggeized particle interactions can be considered as a phenomenological trick, but such inclusion does not have predictive power. The additional important remark to the approach used in Ref. [22]: the contribution of the LO process $q^{\star} \bar{q}^{\star} \rightarrow \gamma(Q \bar{Q} \rightarrow \gamma)$ was ignored. We see that this contribution is dominant and the comparison of theoretical predictions with data without taking it into account is ungrounded.

The Compton scattering of photons on Reggeized quarks,

$$
\gamma+Q \rightarrow \gamma+q,
$$

is a NLO process in the QMRK approach. To calculate it we need to solve the same problems as for other NLO Reggeon-Reggeon to particle-particle processes in the QMRK approch. Firstly, it is necessary to obtain the relevant effective vertex. This task has not been solved yet. Of course, the relevant process is LO if we study the associated photon plus jet, both with large transverse momenta production. However, in that case the effective vertex should be constructed too.

\section{CONCLUSION}

It is shown that it is possible to describe data in the LO QMRK approach for prompt photon spectra for the inclusive photoproduction in the high-energy $\gamma p$ collisions at the HERA Collider. The used here scheme of a calculation have been suggested recently in our

paper [4], in which we described successfully the deep inelastic structure functions $F_{2}$ and $F_{L}$ of the lepton-proton scattering and prompt photon spectra for the inclusive production in the $p \bar{p}$ collisions at the Tevatron Collider. Our results demonstrate that the quark Reggeization hypothesis is a very powerful tool in the high energy phenomenology for the hard processes involving quark exchanges.

\section{ACKNOWLEDGEMENTS}

We thank L. Lipatov and M. Ryskin for the critical remarks and useful discussion of the questions under consideration in this paper. We also thank A. Shipilova for help in numerical 
calculation of unintegrated structure functions in a photon, using KMR prescription.

[1] V. S. Fadin and L. N. Lipatov, Nucl. Phys. B477, 767 (1996); Nucl. Phys. B406, 259 (1993).

[2] L. V. Gribov, E. M. Levin, and M. G. Ryskin, Phys. Rept. 100, 1 (1983); J. C. Collins and R. K. Ellis, Nucl. Phys. B360, 3 (1991); S. Catani, M. Ciafoloni, and F. Hautmann, Nucl. Phys. B366, 135 (1991).

[3] B. A. Kniehl, V. A. Saleev, and D. V. Vasin, Phys. Rev. D 73, 074022 (2006); Phys. Rev. D 74, 014024 (2006); V. A. Saleev and D. V. Vasin, Phys. Rev. D 68, 114013 (2003); V. A. Saleev, Phys. Rev. D 65, 054041 (2002).

[4] V. A. Saleev, Phys. Rev. D 78, 034033 (2008).

[5] L. N. Lipatov, Nucl. Phys. B452, 369 (1995).

[6] V. S. Fadin, M. I. Kotsky, and L. N. Lipatov, Phys. Lett. B 415, 97 (1997); D. Ostrovsky, Phys. Rev. D 62, 054028 (2000); V. S. Fadin, M. G. Kozlov, and A. V. Reznichenko. Report No. DESY 03-025 (2003); J. Bartels, A. S. Vera, and F. Schwennsen, JHEP 0611: 051 (2006).

[7] V. S. Fadin and V. E. Sherman, JETP Lett. 23, 548 (1976); JETP 45, 861 (1977).

[8] ZEUS Collaboration, J. Breitweg et al., Phys. Lett. B 472, 175 (2000).

[9] H1 Collaboration, A. Aktas et al., Eur. Phys. J. C 38, 437 (2005).

[10] M. A. Kimber, A. D. Martin, and M. G. Ryskin, Phys. Rev. D 63, 114027 (2001).

[11] A. D. Martin, R. G. Roberts, W. J. Stirling, and R. S. Thorne, Phys. Lett. B 531, 216 (2002).

[12] M. Glück, E. Reya, and A. Vogt Phys. Rev. D, 46, 1973 (1992).

[13] E. A. Kuraev, L. N. Lipatov, and V. S. Fadin, Sov. Phys. JETP 44, 443 (1976) [Zh. Eksp. Teor. Fiz. 71, 840 (1976)]; I. I. Balitsky and L. N. Lipatov, Sov. J. Nucl. Phys. 28, 822 (1978) [Yad. Fiz. 28, 1597 (1978)].

[14] E. N. Antonov, L. N. Lipatov, E. A. Kuraev, and I. O. Cherednikov, Nucl. Phys. B 721, 111 (2005).

[15] L. N. Lipatov and M. I. Vyazovsky, Nucl. Phys. B 597, 399 (2001).

[16] L. N. Lipatov and V. S. Fadin, JETP Lett. 49, 352 (1989); Sov. J. Nucl. Phys. 50, 712 (1989).

[17] M. Gell-Mann, M. L. Goldberger, F. E. Low, E. Marx, and F. Zachariasen, Phys. Rev. 133, 145B (1964).

[18] V. S. Fadin and R. Fiore, Phys. Rev. D 64, 114012 (2001). 
[19] A. V. Bogdan and V. S. Fadin, Nucl. Phys. B 740, 36 (2006).

[20] D. W. Duke and J. F. Owens, Phys. Rev. D 26, 1600 (1982); J. F. Owens, Rev. Mod. Phys. 59, 465 (1987).

[21] M. Fontannaz, J.P.Guillet and G.Heinrich, Eur. Phys. J. C 21, 303 (2001).

[22] A. V. Lipatov and N. P. Zotov, Phys. Rev. D 72, 054002 (2005). 


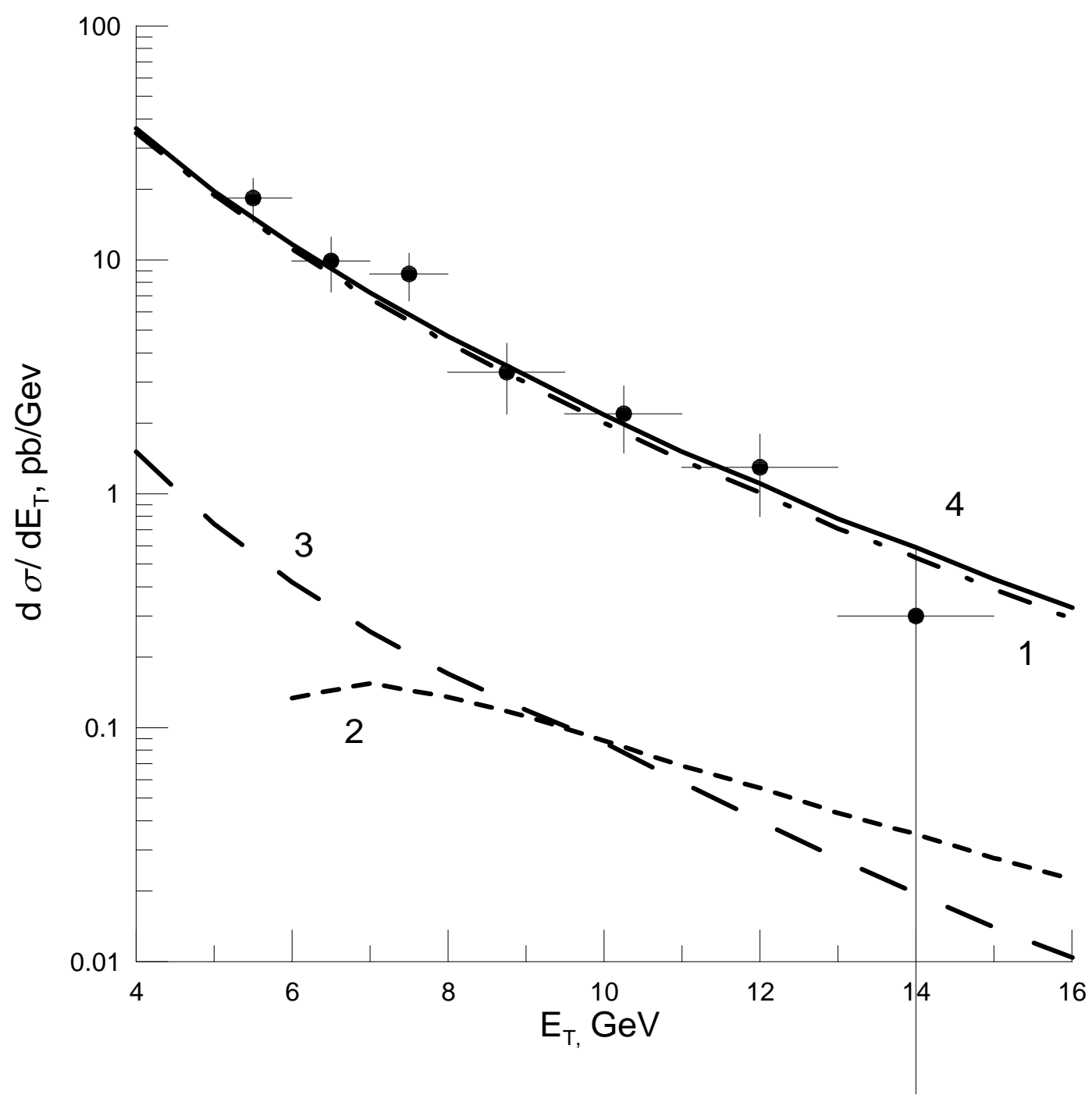

FIG. 1: The $E_{T}$-spectrum of prompt photon at $\sqrt{S}=300 \mathrm{GeV},-0.7<\eta<0.9$, and $0.2<y<0.9$. The curve 1 is the resolved-direct production, 2 is the direct-fragmentation production, 3 is the resolved-fragmentation production, and 4 is their sum. The data are from ZEUS Collaboration [8]. 


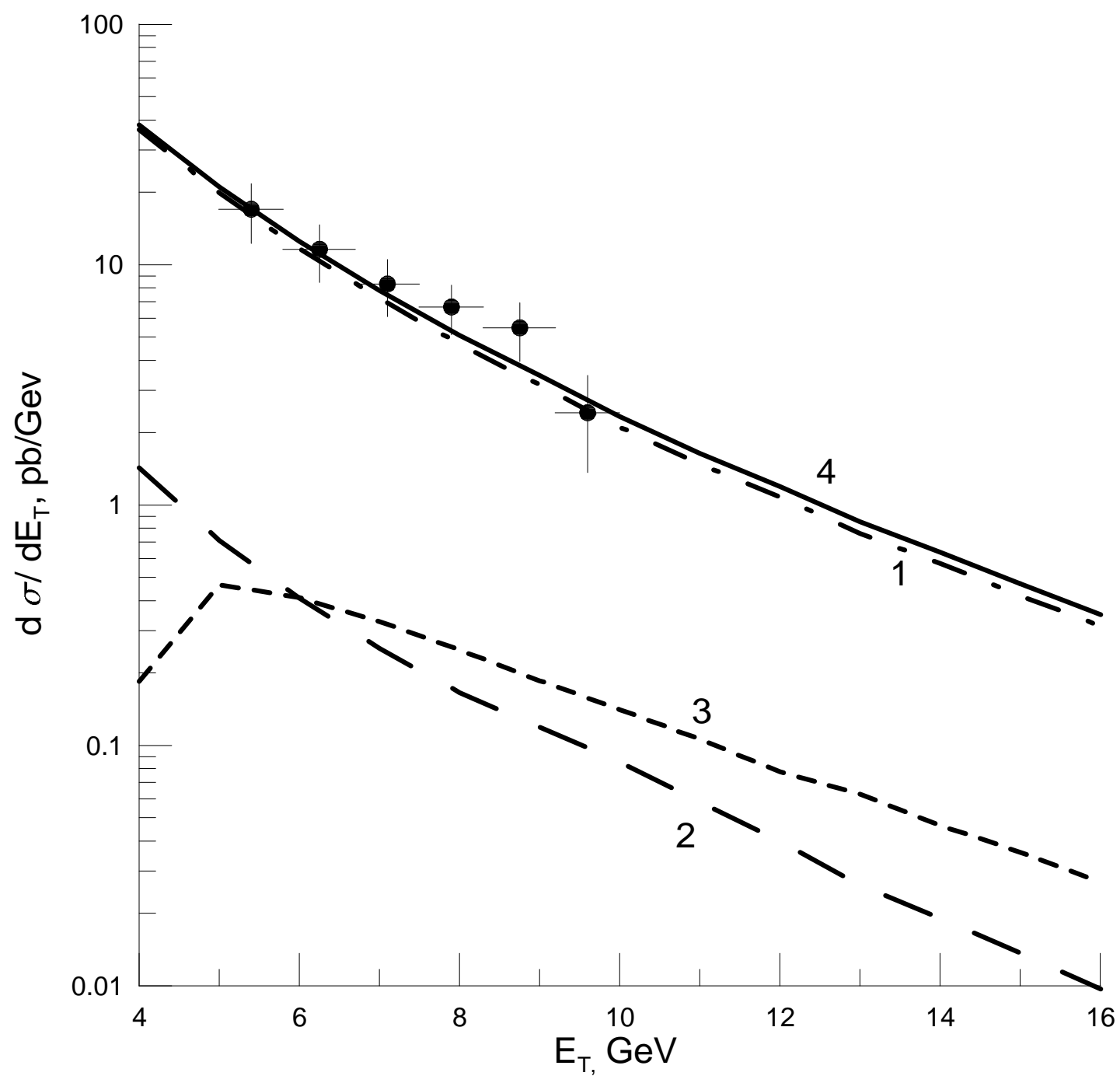

FIG. 2: The $E_{T}$-spectrum of prompt photon at $\sqrt{S}=319 \mathrm{GeV},-1.0<\eta<0.9$, and $0.2<y<0.7$. The curve 1 is the resolved-direct production, 2 is the direct-fragmentation production, 3 is the resolved-fragmentation production, and 4 is their sum. The data are from H1 Collaboration [9]. 


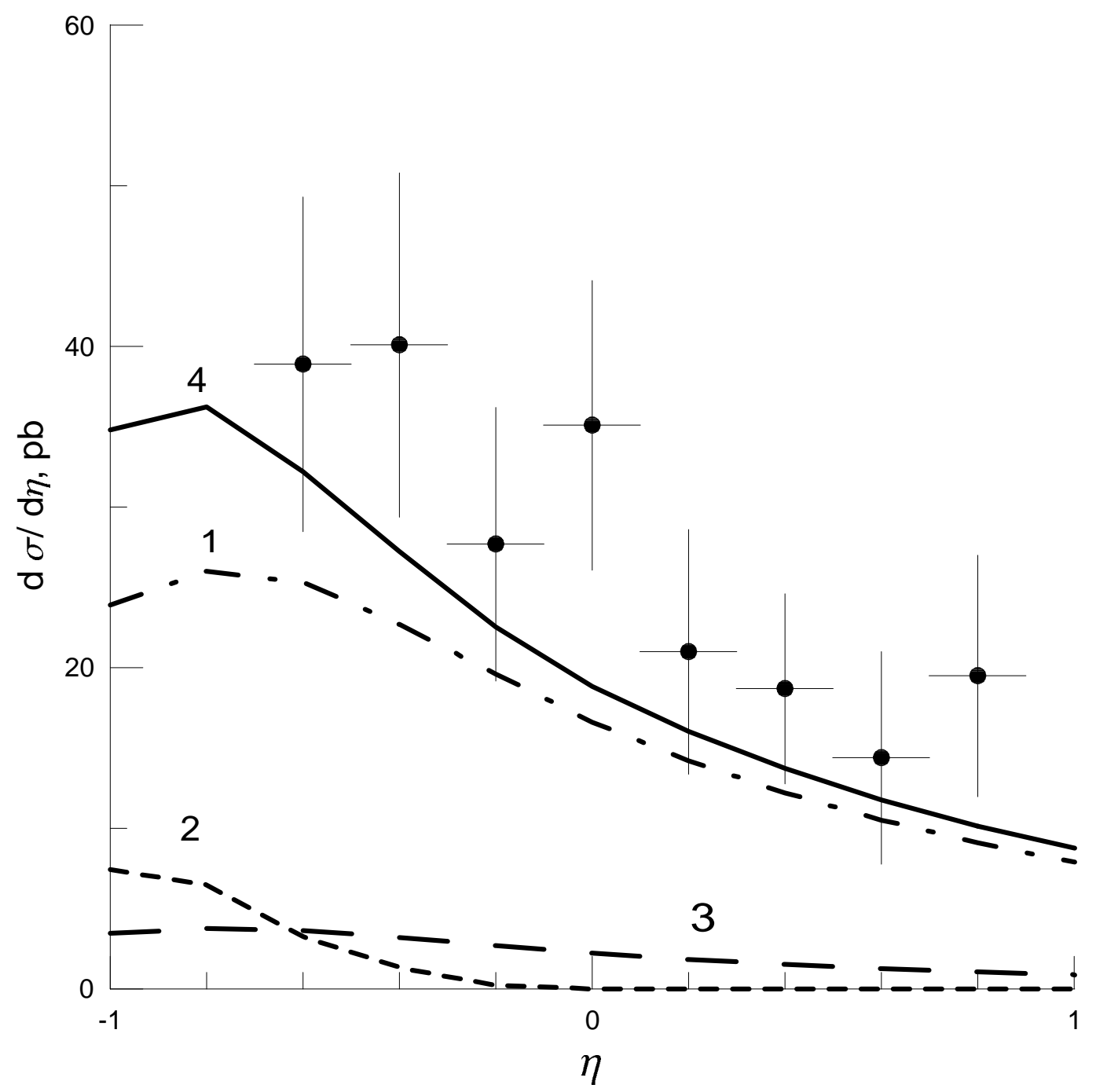

FIG. 3: The $\eta$-spectrum of prompt photon at $\sqrt{S}=300 \mathrm{GeV}, 5<E_{T}<10 \mathrm{GeV}$, and $0.2<y<$ 0.9. The curve 1 is the resolved-direct production, 2 is the direct-fragmentation production, 3 is the resolved-fragmentation production, and 4 is their sum. The data are from ZEUS Collaboration [8]. 


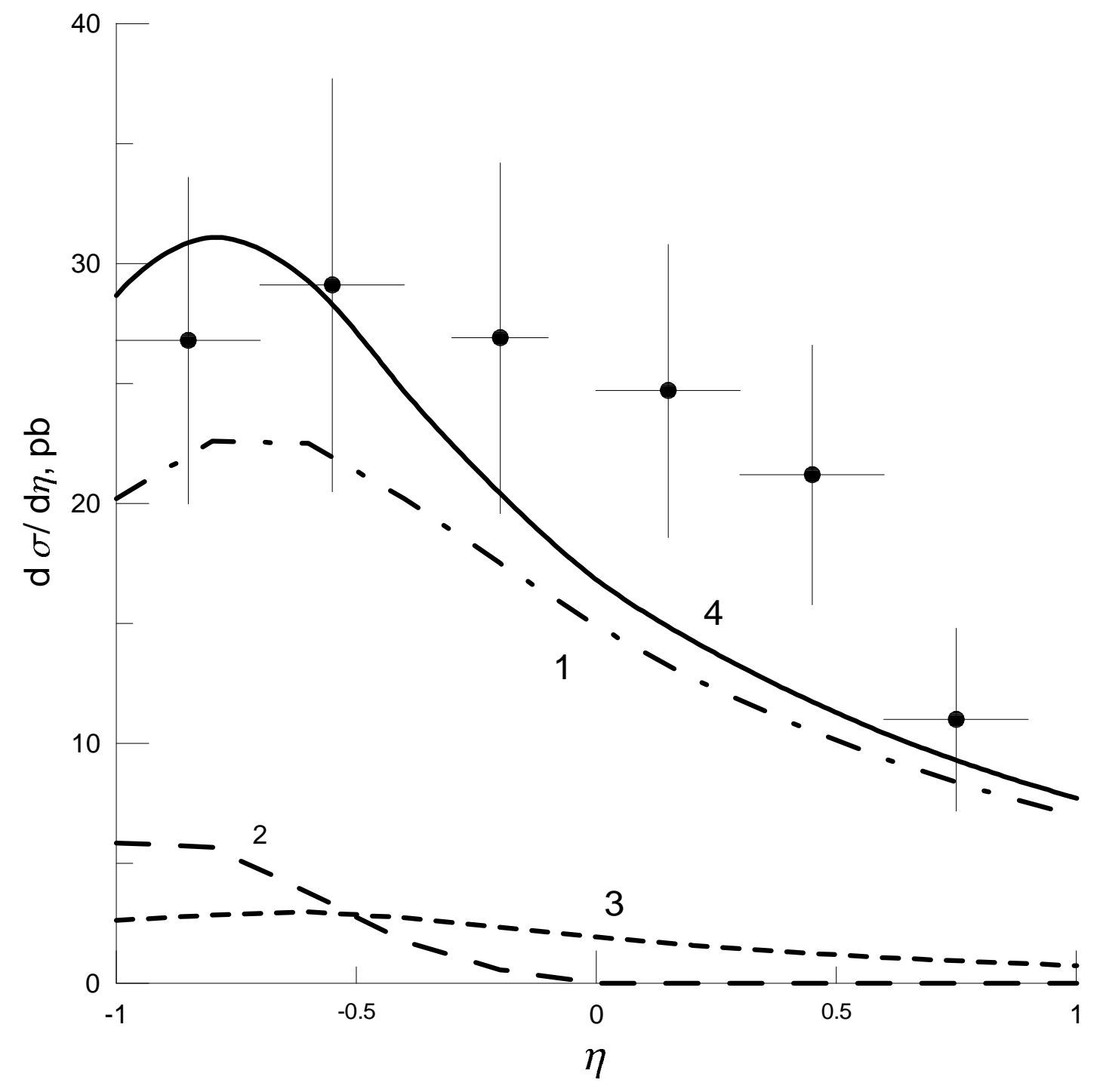

FIG. 4: The $\eta$-spectrum of prompt photon at $\sqrt{S}=319 \mathrm{GeV}, 5<E_{T}<10 \mathrm{GeV}$, and $0.2<y<$ 0.7. The curve 1 is the resolved-direct production, 2 is the direct-fragmentation production, 3 is the resolved-fragmentation production, and 4 is their sum. The data are from H1 Collaboration 9]. 